\title{
Improved results on nonlinear perturbed T-S fuzzy systems with interval time-varying delays using a geometric sequence division method
}

\author{
Hao Chen ${ }^{*}$
}

*Correspondence:

haochen84@yahoo.co.uk

College of Electrical

and Information

Engineering, Southwest

University for Nationalities,

Chengdu 610041, China

\begin{abstract}
This paper presents improved stability results by introducing a new delay partitioning method based on the theory of geometric progression to deal with T-S fuzzy systems in the appearance of interval time-varying delays and nonlinear perturbations. A common ratio $\alpha$ is applied to split the delay interval into multiple unequal subintervals. A modified Lyapunov-Krasovskii functional (LKF) is constructed with triple-integral terms and augmented factors including the length of every subintervals. In addition, the recently developed free-matrix-based integral inequality is employed to combine with the extended reciprocal convex combination and free weight matrices techniques for avoiding the overabundance of the enlargement when deducing the derivative of the LKF. Eventually, this developed research work can efficiently obtain the maximum upper bound of the time-varying delay with much less conservatism. Numerical results are conducted to illustrate the remarkable improvements of this proposed method.
\end{abstract}

Keywords: Delay-partitioning, Geometric sequence division, Interval time-varying delays, Nonlinear perturbations, T-S fuzzy systems

\section{Background}

Over the past few decades, complex mathematical modelling with higher order is frequently encountered in many engineering applications, which may cause nonlinearity in dynamic systems. The T-S fuzzy theory introduced in Takagi and Sugeno (1985) can be flexibly applied to approximate the complex nonlinear systems into a unified framework (Zeng et al. 2014; Chang et al. 2015; Chang and Wang 2015; Balasubramaniam et al. 2012; Tanaka et al. 2003). Due to material transfer requirement, accumulation of time lags through system connections and process time, time delays commonly exist in dynamics systems such as chemical processes, communication networks and biological systems, which is considered as a source of instability. Stability analysis of time delayed T-S fuzzy systems has thus been paid special attention (Wu et al. 2011; Zhang et al. 2015b; Zhao et al. 2009).

Stability conditions are classified into two categories delay-independent and delaydependent. As much of information on the delay is concerned, the delay-dependent

C 2016 The Author(s). This article is distributed under the terms of the Creative Commons Attribution 4.0 International License (http://creativecommons.org/licenses/by/4.0/), which permits unrestricted use, distribution, and reproduction in any medium, provided you give appropriate credit to the original author(s) and the source, provide a link to the Creative Commons license, and indicate if changes were made. 
criteria is more useful to produce less conservative results (Yang et al. 2015b; Senthilkumar and Mahanta 2010; Lam et al. 2007). Delay partitioning technique, alternatively known as a delay fractionizing method, was developed in Gouaisbaut and Peaucelle (2006). A number of research works have been developed to prove that delay partitioning approach can significantly enhance the stability conditions to obtain less conservatism as soon as the partitions get thinner (Yang et al. 2015a; Zhao et al. 2009; Wang et al. 2015). In Wang et al. (2015), a secondary partitioning method was proposed to further divide primarily separated intervals into a series of smaller segments, which illustrates good stability results. Nonetheless, the research development requires too many adjustable parameters. It thus cost extra computation burden.

In order to further achieve less conservative results, a number of inequalities methods have been proposed, such as Peng-Park's inequality, reciprocally convex combination, free-matrix-based inequality, etc, which are employed for the purpose of overabundance reduction of the enlargement of the Lyapunov functionals derivative (Sun et al. 2010; Gyurkovics 2015; Park et al. 2011, 2015; Peng and Han 2011; Zeng et al. 2015a). By introducing both augmented state and integral of the state over the period of the delay, these newly developed techniques can preserve extra items when dealing with the enlargement in bounding the derivative of the LKF comparing to the Jensen's inequality in Seuret and Gouaisbaut (2013). As a result, tighter bounding inequalities are obtained to reduce the conservatism.

In addition, the presence of nonlinearity can cause poor performance and even instability in engineering systems. Robust stability analysis with the effect of the nonlinear perturbation has been investigated with considerable attention (Zhang et al. 2010, 2015a; Ramakrishnan and Ray 2011). Because of process uncertainties and parameter variations, nonlinear perturbations commonly occur in both current and delayed states Ramakrishnan and Ray (2011). The previously developed techniques for such systems are rarely adaptive for the stability analysis with the appearance of nonlinear perturbations. In this paper, T-S fuzzy systems with interval time-varying delays and nonlinear perturbation are considered for stability analysis. Based on the geometric sequence division, some newly developed inequalities, free weight matrices techniques and the Finsler's Lemma are also employed for obtaining improved stability criteria. Main contributions of this work are:

1. Based on the recently developed geometric sequence division method on delay partitioning, improved stability criteria is presented.

2. Extended reciprocal convex combination(ERCC) is employed for the less enlargement of bounding the derivative of the augmented LKF which is able to reduce the overabundance when deal with the inequalities in the derivative of the LKF.

3. In terms of the system equation, free weight matrices techniques are applied to reduce the conservatism with respect to each fuzzy rule. Numerical examples are conducted to show that the improved stability conditions are obtained by comparing with some existing results.

Notations. $\mathbb{R}^{n}$ and $\mathbb{R}^{n \times m}$ denote the $\mathrm{n}$-dimensional Euclidean space and the set of all $n \times m$ real matrices, respectively. $I(0)$ is the identity (zero) matrix with appropriate 
dimension; $A^{\mathrm{T}}$ denotes the transpose, and $\operatorname{He}(A)=A+A^{\mathrm{T}}$. The symbol $*$ denotes the elements below the main diagonal of a symmetric block matrix. $\|\bullet\|$ is the Euclidean norm in $\mathbb{R}^{n}$. $C\left(\left[-\tau_{b}, 0\right], \mathbb{R}^{n}\right)$ is the family of continuous functions $\varphi$ from the interval $\left[-\tau_{b}, 0\right]$ to $\mathbb{R}^{n}$ with the norm $\|\varphi\|_{\tau}=\sup _{-\tau \leq \theta \leq 0}\|\varphi(\theta)\|$. The notation $A>(\geq) B$ means that $A-B$ is positive (semi-positive) definite.

\section{Problem statements and preliminaries}

Considering nonlinear perturbed T-S fuzzy systems with interval time-varying delays, for each $l=1,2, \ldots r$ ( $r$ is the number of the plant rules), the $l$ th rule of this fuzzy model with $r$ plant rules are described as follows.

Rule $l$ : IF $z_{1}(t)$ is $M_{l 1}$ and $\cdots z_{p}(t)$ is $M_{l p}$ THEN

$$
\begin{aligned}
& \dot{x}(t)=A_{l} x(t)+B_{l} x(t-\tau(t))+C_{l} f(x(t), t)+D_{l} g(x(t-\tau(t)), t), \quad t \geq 0 \\
& x(t)=\varphi(t), \quad t \in\left[-\tau_{b}, 0\right]
\end{aligned}
$$

where $x(t) \in \mathbb{R}^{n}$ is the state variable, $z_{s}(t), M_{l s}(s=1,2, \ldots, p)$ are premise variables and the related fuzzy sets, respectively. $A_{l}, B_{l}, C_{l}, D_{l}$ are the constant matrices with appropriate dimensions. $\tau(t)$ is the time-varying delay. $f(x(t), t)$ and $g(x(t-\tau(t)), t)$ are unknown nonlinear perturbations with respect to the current state $x(t)$ and the delayed state $x(t-\tau(t))$. $\varphi(t) \in C\left(\left[-\tau_{b}, 0\right], \mathbb{R}^{n}\right)$ is the initial function.

Then the fuzzy model can be inferred as:

$$
\begin{aligned}
\dot{x}(t) & =\sum_{l=1}^{r} h_{l}(t)\left[A_{l} x(t)+B_{l} x(t-\tau(t))+C_{l} f(x(t), t)+D_{l} g(x(t-\tau(t)), t)\right] \\
& =A(t) x(t)+B(t) x(t-\tau(t))+C(t) f(x(t), t)+D(t) g(x(t-\tau(t)), t), \quad t \geq 0 \\
x(t) & =\varphi(t), \quad t \in\left[-\tau_{b}, 0\right]
\end{aligned}
$$

where $r$ is the number of fuzzy implications, $h_{l}(t)=\frac{W_{l}(t)}{\sum_{l=1}^{r} W_{l}(t)}, W_{l}(t)=\prod_{s=1}^{p} M_{l s}\left(z_{s}(t)\right)$ with $M_{l s}\left(z_{s}(t)\right)$ is the grade of membership of $z_{s}(t)$ in $M_{l s} . A(t)=\sum_{l=1}^{r} h_{l}(t) A_{l}$, $B(t)=\sum_{l=1}^{r} h_{l}(t) B_{l} \quad, \quad C(t)=\sum_{l=1}^{r} h_{l}(t) C_{l}, \quad D(t)=\sum_{l=1}^{r} h_{l}(t) D_{l} . \quad$ For $\quad W_{l}(t) \geq 0$, $h_{l}(t) \geq 0$ and $\sum_{l=1}^{r} h_{l}(t)=1$ thus holds.

The time-varying delay $\tau(t)$ is considered as the following two cases:

Case $1 \tau(t)$ is a differentiable function satisfying

$$
0 \leq \tau_{a} \leq \tau(t) \leq \tau_{b}, \dot{\tau}(t)<\mu, \quad \forall t \geq 0,
$$

Case $2 \tau(t)$ is a continuous function satisfying

$$
0 \leq \tau_{a} \leq \tau(t) \leq \tau_{b}, \quad \forall t \geq 0,
$$

where $\tau_{a}, \tau_{b}, \mu$ are constants.

Assumption $1 \quad f(0, t) \equiv 0, g(0, t) \equiv 0$ and

$$
\left\{\begin{array}{l}
f^{\mathrm{T}} f \leq \gamma^{2} x^{\mathrm{T}}(t) F^{\mathrm{T}} F x(t) \\
g^{\mathrm{T}} g \leq \beta^{2} x^{\mathrm{T}}(t-\tau(t)) G^{\mathrm{T}} G x(t-\tau(t))
\end{array}\right.
$$


where $\gamma \geq 0, \beta \geq 0$ are known scalars, $F$ and $G$ are known constant matrices, $\forall x \in \mathbb{R}^{n}$, and $f$ and $g$ are the short expressions of $f(x(t), t)$ and $g(x(t-\tau(t)), t)$, respectively.

A few lemmas are introduced for stability analysis as follows.

Lemma 1 (Han 2003, 2005) For $n \times n$ matrix $Q>0$, scalar $\tau>0$, vector-valued function $\dot{x}:[-\tau, 0] \longrightarrow \mathbb{R}^{n}$ such that the following integrations are well defined, it holds that

$$
\begin{gathered}
-\tau \int_{t-\tau}^{t} \dot{x}^{\mathrm{T}}(s) Q \dot{x}(s) \mathrm{d} s \leq\left[x^{\mathrm{T}}(t) x^{\mathrm{T}}(t-\tau)\right]\left[\begin{array}{cc}
-Q & Q \\
* & -Q
\end{array}\right]\left[\begin{array}{c}
x(t) \\
x(t-\tau)
\end{array}\right] \\
-\frac{\tau^{2}}{2} \int_{-\tau}^{0} \int_{t+\theta}^{t} \dot{x}^{\mathrm{T}}(s) Q \dot{x}(s) \mathrm{d} s \mathrm{~d} \theta \leq\left[\tau x^{\mathrm{T}}(t) \int_{t-\tau}^{t} x^{\mathrm{T}}(s) \mathrm{d} s\right]\left[\begin{array}{cc}
-Q & Q \\
* & -Q
\end{array}\right]\left[\begin{array}{c}
\tau x(t) \\
\int_{t-\tau}^{t} x(s) \mathrm{d} s
\end{array}\right]
\end{gathered}
$$

Lemma 2 (Zeng et al. 2015, Free-matrix-based integral inequality) Let $x$ be a differentiable function $:[a, b] \rightarrow \mathbb{R}^{n}, Z \in \mathbb{R}^{n \times n}$ and $W_{1}, W_{3} \in \mathbb{R}^{3 n \times 3 n}$ be symmetric matrices, and $W_{2} \in \mathbb{R}^{3 n \times 3 n}, N_{1}, N_{2} \in \mathbb{R}^{3 n \times n}$ satisfying this condition

$$
\left[\begin{array}{ccc}
W_{1} & W_{2} & N_{1} \\
* & W_{3} & N_{2} \\
* & * & Z
\end{array}\right] \geq 0
$$

it holds:

$$
-\int_{a}^{b} \dot{x}^{\mathrm{T}}(s) Z \dot{x}(s) \mathrm{d} s \leq \varpi^{\mathrm{T}} \Omega \varpi
$$

whereळ $=\left[x^{\mathrm{T}}(b) x^{\mathrm{T}}(a) \frac{1}{b-a} \int_{a}^{b} x^{\mathrm{T}}(s) \mathrm{d} s\right]^{\mathrm{T}}, \Omega=(b-a)\left(W_{1}+\frac{1}{3} W_{3}\right)+\operatorname{He}\left(N_{1} \Lambda_{1}+N_{2} \Lambda_{2}\right)$, $\Lambda_{1}=\bar{e}_{1}-\bar{e}_{2}, \Lambda_{2}=2 \bar{e}_{3}-\bar{e}_{1}-\bar{e}_{2}, \bar{e}_{1}=\left[\begin{array}{lll}I & 0 & 0\end{array}\right], \bar{e}_{2}=\left[\begin{array}{lll}0 & I & 0\end{array}\right], \bar{e}_{3}=\left[\begin{array}{lll}0 & 0 & I\end{array}\right]$.

Remark 1 By introducing both augmented state and integral of the state over the period of the delay, the well known Wirtinger-based inequality was developed with less conservatism comparing to the Jensen's inequality in Seuret and Gouaisbaut (2013) to reduce enlargement in bounding the derivative of the LKF inequalities. However, due to the unadjustable parameters, the tightest upper bound is rarely to be determined in this development. In fact, this Wirtinger-based inequality is the special case of free-matrixbased integral inequality ( 8 ) by setting

$$
\begin{aligned}
& N_{1}=\frac{1}{b-a}\left[\begin{array}{lll}
-Z & Z & 0
\end{array}\right], N_{2}=\frac{3}{b-a}\left[\begin{array}{lll}
Z & Z & -2 Z
\end{array}\right], \\
& W_{1}=N_{1} Z^{-1} N_{1}^{\mathrm{T}}, W_{2}=N_{1} Z^{-1} N_{2}^{\mathrm{T}}, W_{3}=N_{2} Z^{-1} N_{2}^{\mathrm{T}}
\end{aligned}
$$

Particularly, a set of slack variables inequality in this inequality can be flexibly adjusted, which provide remarkable extra freedom for the purpose of conservatism reduction.

Lemma 3 (Wang et al. 2015, Extendedreciprocalconvexcombination-ERCC) For any vectors $f_{1}, \ldots, f_{N}$ with appropriate dimensions, scalars $k_{i}(t) \in[0,1], \sum_{i=1}^{N} k_{i}(t)=1$, and matrices $R_{i}>0$, there exist matrix $S_{i j}(i=1, \ldots, N-1, j=i+1, \ldots, N)$ satisfies 


$$
\left[\begin{array}{ll}
R_{i} & S_{i j} \\
* & R_{j}
\end{array}\right] \geq 0
$$

then the following inequality holds:

$$
-\sum_{i=1}^{N} \frac{1}{k_{i}(t)} f_{i}^{\mathrm{T}} R_{i} f_{i} \leq-\left[\begin{array}{l}
f_{1} \\
\vdots \\
f_{N}
\end{array}\right]^{\mathrm{T}}\left[\begin{array}{lll}
R_{1} & \cdots & S_{1, N} \\
* & \ddots & \vdots \\
* & * & R_{N}
\end{array}\right]\left[\begin{array}{l}
f_{1} \\
\vdots \\
f_{N}
\end{array}\right]
$$

Lemma 4 (de Oliveira and Skelton 2001, Finsler's Lemma) Let $\zeta \in \mathbb{R}^{n}, \Phi=\Phi^{\mathrm{T}} \in \mathbb{R}^{n \times n}$, and $B \in \mathbb{R}^{m \times n}$ with rank $(B)<n$. The following statements are equivalent:

(i) $\quad \zeta^{\mathrm{T}} \Phi \zeta<0, \forall B \zeta=0, \quad \zeta \neq 0$;

(ii) $\quad B^{\perp^{\mathrm{T}}} \Phi B^{\perp}<0$;

(iii) $\exists \mathcal{L} \in \mathbb{R}^{n \times m}: \Phi+\operatorname{He}(\mathcal{L} B)<0$;

where $B^{\perp} \in \mathbb{R}^{n \times(n-\operatorname{rank}(B))}$ is the right orthogonal complement of $B$.

\section{Main results}

The stability criteria of T-S fuzzy systems in the presences of interval time-varying delays and nonlinear perturbations are analyzed in this section. In terms of the geometric sequence division method, a new delay partitioning technique is proposed in Fig. 1.

For any integral $m=q-1 \geq 1$, the delay interval $\left[\tau_{a}, \tau_{b}\right]$ is separated into $m$ unequal geometric subintervals as,

$$
\left\{\begin{array}{l}
\delta_{i}=\alpha^{q-i} \\
\tau_{i}=\tau_{0}+\sum_{k=1}^{i} \alpha^{q-k}, \quad i=1,2, \cdots, q-1
\end{array}\right.
$$

where $\tau_{a}=\tau_{0}, \tau_{b}=\tau_{q-1}$, and $m$ is the number of segments of interval $\left[\tau_{a}, \tau_{b}\right]$. It is expressed as $\left[\tau_{a}, \tau_{b}\right]=\left[\tau_{0}, \tau_{1}\right] \bigcup_{i=2}^{m}\left(\tau_{i-1}, \tau_{i}\right] \triangleq I_{1} \cup I_{2} \cup \ldots \cup I_{m} . \alpha$ is a real positive number, and $\delta_{i}$ is the length of the ith subinterval which equals to $\alpha^{q-i}$. The following expressions are used for notational simplification.

$$
e_{j}=[\underbrace{0, \cdots, 0}_{j-1}, I \underbrace{0, \cdots, 0}_{3 m-j+6}]^{\mathrm{T}} \in \mathbb{R}^{n \times(3 m+6) n}, \quad j=1,2, \cdots, 3 m+6
$$

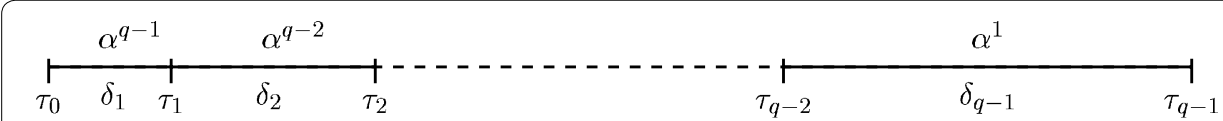

Fig. 1 Geometric sequence division based delay partitioning 
The augmented vector is defined as,

$$
\xi(t)=\left[\dot{x}^{\mathrm{T}}(t), x^{\mathrm{T}}(t), x^{\mathrm{T}}\left(t-\tau_{0}\right), \eta^{\mathrm{T}}(t), x^{\mathrm{T}}(t-\tau(t)), \eta_{1}^{\mathrm{T}}(t), \eta_{2}^{\mathrm{T}}(t), f^{\mathrm{T}}, g^{\mathrm{T}}\right]^{\mathrm{T}}
$$

where

$$
\begin{aligned}
\eta(t) & =\left[x^{\mathrm{T}}\left(t-\tau_{1}\right), \cdots, x^{\mathrm{T}}\left(t-\tau_{q-1}\right)\right]^{\mathrm{T}} \\
\eta_{1}(t) & =\left[\int_{t-\tau_{0}}^{t} x^{\mathrm{T}}(s) \mathrm{d} s, \cdots, \int_{t-\tau_{q-2}}^{t} x^{\mathrm{T}}(s) \mathrm{d} s\right]^{\mathrm{T}} \\
\eta_{2}(t) & =\left[\frac{1}{\delta_{1}} \int_{t-\tau_{1}}^{t-\tau_{0}} x^{\mathrm{T}}(s) \mathrm{d} s, \cdots, \frac{1}{\delta_{q-1}} \int_{t-\tau_{q-1}}^{t-\tau_{q-2}} x^{\mathrm{T}}(s) \mathrm{d} s\right]^{\mathrm{T}}
\end{aligned}
$$

Next, the new delay dependent stability criteria is presented for the T-S fuzzy system described in (2).

Theorem 1 Given a positive integer $m$, and $\delta_{i}=\alpha^{q-i}$. Consider (3) with time-varying delay satisfying Case 1. The system (2) is asymptotically stable if there exist symmetric positive definite matrices $Z_{i}, Q_{i}, \widetilde{Q}, R_{2 i}, R_{3 i} \in \mathbb{R}^{n \times n}(i=1,2, \ldots, m)$, $\mathcal{P}=\left[P_{i j}\right]_{(m+1) \times(m+1)} \in \mathbb{R}^{(m+1) n \times(m+1) n}$, symmetric matrices $W_{1}, W_{3} \in \mathbb{R}^{3 n \times 3 n}$, and $J \in \mathbb{R}^{n \times n}$, matrices $W_{2} \in \mathbb{R}^{3 n \times 3 n}, N_{1}, N_{2} \in \mathbb{R}^{3 n \times n}$, and $\mathcal{Y} \in \mathbb{R}^{(3 m+6) n \times n}$,such that the following LMIs hold

$$
\begin{aligned}
& W_{i}=\left[\begin{array}{ccc}
W_{1} & W_{2} & N_{1} \\
* & W_{3} & N_{2} \\
* & * & Z_{i}
\end{array}\right] \geq 0 \\
& \Psi_{k, l}+\operatorname{He}\left(\mathcal{Y} \Gamma_{l}\right)<0, \quad l=1,2, \ldots r
\end{aligned}
$$

where

$$
\begin{aligned}
& \Gamma_{l}=A_{l} e_{2}^{\mathrm{T}}+B_{l} e_{m+4}^{\mathrm{T}}+C_{l} e_{3 m+5}^{\mathrm{T}}+D_{l} e_{3 m+6}^{\mathrm{T}}-e_{1}^{\mathrm{T}} \\
& \Psi_{k, l}=\Psi_{1}+\Psi_{2}+\Psi_{3 k}+\Psi_{4}+\Psi_{51}+\Psi_{52}+\Psi_{l, 6}+e_{1} \mathcal{Z} e_{1}^{\mathrm{T}} \\
& \Psi_{1}=\operatorname{He}\left\{\left[\begin{array}{l}
e_{2}^{\mathrm{T}} \\
e_{2 m+5}^{\mathrm{T}} \\
\vdots \\
e_{3 m+4}^{\mathrm{T}}
\end{array}\right]^{\mathrm{T}} \mathcal{P}\left[\begin{array}{l}
e_{1}^{\mathrm{T}} \\
\frac{1}{\delta_{1}}\left(e_{3}^{\mathrm{T}}-e_{4}^{\mathrm{T}}\right) \\
\vdots \\
\frac{1}{\delta_{q-1}}\left(e_{m+2}^{\mathrm{T}}-e_{m+3}^{\mathrm{T}}\right)
\end{array}\right]\right\} \\
& \Psi_{2}=e_{2} \widetilde{Q} e_{2}^{\mathrm{T}}-(1-\mu) e_{m+4} \widetilde{Q} e_{m+4}^{\mathrm{T}} \\
& +\left[\begin{array}{c}
e_{3}^{\mathrm{T}} \\
e_{4}^{\mathrm{T}} \\
\vdots \\
e_{m+2}^{\mathrm{T}} \\
e_{m+3}^{\mathrm{T}}
\end{array}\right]^{\mathrm{T}}\left[\begin{array}{ccccc}
Q_{1} & 0 & \cdots & \cdots & 0 \\
* & Q_{2}-Q_{1} & 0 & \cdots & \vdots \\
* & * & \ddots & 0 & \vdots \\
* & * & * & Q_{m}-Q_{m-1} & 0 \\
* & * & * & * & -Q_{m}
\end{array}\right]\left[\begin{array}{c}
e_{3}^{\mathrm{T}} \\
e_{4}^{\mathrm{T}} \\
\vdots \\
e_{m+2}^{\mathrm{T}} \\
e_{m+3}^{\mathrm{T}}
\end{array}\right]
\end{aligned}
$$




$$
\begin{aligned}
& \Psi_{3 k}=\sum_{i=1, i \neq k}^{q-1}\left[\begin{array}{c}
e_{i+2}^{\mathrm{T}} \\
e_{i+3}^{\mathrm{T}} \\
e_{2 m+4+i}^{\mathrm{T}}
\end{array}\right]^{\mathrm{T}} \Omega_{3}\left[\begin{array}{c}
e_{i+2}^{\mathrm{T}} \\
e_{i+3}^{\mathrm{T}} \\
e_{2 m+4+i}^{\mathrm{T}}
\end{array}\right] \\
& +\left[\begin{array}{c}
e_{k+2}^{\mathrm{T}} \\
e_{m+4}^{\mathrm{T}} \\
e_{k+3}^{\mathrm{T}}
\end{array}\right]^{\mathrm{T}}\left[\begin{array}{ccc}
-Z_{k} & Z_{k}-J & J \\
* & -2 Z_{k}+J^{\mathrm{T}}+J & Z_{k}-J \\
* & * & -Z_{k}
\end{array}\right]\left[\begin{array}{c}
e_{k+2}^{\mathrm{T}} \\
e_{m+4}^{\mathrm{T}} \\
e_{k+3}^{\mathrm{T}}
\end{array}\right] \\
& \Psi_{4}=\sum_{i=1}^{q-1}\left[\begin{array}{c}
\tau_{i-1} e_{2}^{\mathrm{T}} \\
e_{m+4+i}^{\mathrm{T}}
\end{array}\right]^{\mathrm{T}}\left[\begin{array}{ll}
-R_{2 i} & R_{2 i} \\
* & -R_{2 i}
\end{array}\right]\left[\begin{array}{c}
\tau_{i-1} e_{2}^{\mathrm{T}} \\
e_{m+4+i}^{\mathrm{T}}
\end{array}\right] \\
& +\sum_{i=1}^{q-1} \delta_{i}^{2}\left[\begin{array}{l}
e_{2}^{\mathrm{T}} \\
e_{2 m+4+i}^{\mathrm{T}}
\end{array}\right]^{\mathrm{T}}\left[\begin{array}{ll}
-R_{3 i} & R_{3 i} \\
* & -R_{3 i}
\end{array}\right]\left[\begin{array}{l}
e_{2}^{\mathrm{T}} \\
e_{2 m+4+i}^{\mathrm{T}}
\end{array}\right] \\
& \Psi_{51}=e_{2} \lambda_{1} \gamma^{2} F^{\mathrm{T}} F e_{2}^{\mathrm{T}}-e_{3 m+5} \lambda_{1} I e_{3 m+5}^{\mathrm{T}} \\
& \Psi_{52}=e_{m+4} \lambda_{2} \beta^{2} G^{\mathrm{T}} G e_{m+4}^{\mathrm{T}}-e_{3 m+6} \lambda_{2} I e_{3 m+6}^{\mathrm{T}}
\end{aligned}
$$

$$
\Psi_{l, 6}=\left[\begin{array}{l}
e_{1}^{\mathrm{T}} \\
e_{2}^{\mathrm{T}} \\
e_{m+4}^{\mathrm{T}} \\
e_{3 m+5}^{\mathrm{T}} \\
e_{3 m+6}^{\mathrm{T}}
\end{array}\right]^{\mathrm{T}}\left[\begin{array}{lllll}
-\widehat{N}_{1}-\widehat{N}_{1}^{\mathrm{T}} & \widehat{N}_{1} A_{l}-\widehat{N}_{2}^{\mathrm{T}} & \widehat{N}_{1} B_{l} & \widehat{N}_{1} C_{l} & \widehat{N}_{1} D_{l} \\
* & \widehat{N}_{2} A_{l}+A_{l}^{\mathrm{T}} \widehat{N}_{2}^{\mathrm{T}} & \widehat{N}_{2} B_{l} & \widehat{N}_{2} C_{l} & \widehat{N}_{2} D_{l} \\
* & * & 0 & 0 & 0 \\
* & * & 0 & 0 & 0 \\
* & * & 0 & 0 & 0
\end{array}\right]\left[\begin{array}{l}
e_{1}^{\mathrm{T}} \\
e_{2}^{\mathrm{T}} \\
e_{m+4}^{\mathrm{T}} \\
e_{3 m+5}^{\mathrm{T}} \\
e_{3 m+6}^{\mathrm{T}}
\end{array}\right]
$$

with

$$
\begin{aligned}
\mathcal{Z} & =\sum_{i=1}^{q-1} \delta_{i} \alpha^{q-i} Z_{i}+\sum_{i=1}^{q-1} \frac{1}{4} \tau_{i-1}^{4} R_{2 i}+\sum_{i=1}^{q-1} \frac{1}{4}\left(\tau_{i}^{2}-\tau_{i-1}^{2}\right)^{2} R_{3 i} \\
\Omega_{3} & =\delta_{i}^{2}\left(W_{1}+\frac{1}{3} W_{3}\right)+\delta_{i} \operatorname{He}\left(N_{1} \Lambda_{1}+N_{2} \Lambda_{2}\right)
\end{aligned}
$$

Proof For any $t \geq 0$, there should exist an integer $k \in\{1,2, \ldots, m\}$, such that $\tau(t) \in I_{k}$. The Lyapunov-Krasovskii functional is as follows:

$$
\left.V\left(x_{t}, k\right)\right|_{\tau(t) \in I_{k}}=V_{1}\left(x_{t}\right)+V_{2}\left(x_{t}\right)+V_{3}\left(x_{t}, k\right)+V_{4}\left(x_{t}\right)
$$


where

$$
\begin{aligned}
V_{1}\left(x_{t}\right) & =\epsilon^{\mathrm{T}}(t) \mathcal{P} \epsilon(t) \\
V_{2}\left(x_{t}\right) & =\int_{t-\tau(t)}^{t} x^{\mathrm{T}}(s) \widetilde{Q} x(s) \mathrm{d} s+\sum_{i=1}^{q-1} \int_{t-\tau_{i}}^{t-\tau_{i-1}} x^{\mathrm{T}}(t) Q_{i} x(t) \mathrm{d} s \\
V_{3}\left(x_{t}, k\right) & =\sum_{i=1}^{q-1} \delta_{i} \int_{-\tau_{i}}^{-\tau_{i-1}} \int_{t+\beta}^{t} \dot{x}^{\mathrm{T}}(s) Z_{i} \dot{x}(s) \mathrm{d} s \mathrm{~d} \beta \\
V_{4}\left(x_{t}\right) & =\sum_{i=1}^{q-1} \frac{\tau_{i-1}^{2}}{2} \int_{-\tau_{i-1}}^{0} \int_{\theta}^{0} \int_{t+\lambda}^{t} \dot{x}^{\mathrm{T}}(s) R_{2 i} \dot{x}(s) \mathrm{d} s \mathrm{~d} \lambda \mathrm{d} \theta \\
& +\sum_{i=1}^{q-1} \frac{\tau_{i}^{2}-\tau_{i-1}^{2}}{2} \int_{-\tau_{i}}^{-\tau_{i-1}} \int_{\theta}^{0} \int_{t+\lambda}^{t} \dot{x}^{\mathrm{T}}(s) R_{3 i} \dot{x}(s) \mathrm{d} s \mathrm{~d} \lambda \mathrm{d} \theta
\end{aligned}
$$

with $\epsilon(t)=\left[x^{\mathrm{T}}(t), \eta_{2}^{\mathrm{T}}(t)\right]^{\mathrm{T}}$

The derivative of the Lyapunov functional $\left.V\left(x_{t}, k\right)\right|_{\tau(t) \in I_{k}}$ along the trajectory of the perturbed T-S fuzzy system described in (2) is given as:

$$
\left.\dot{V}\left(x_{t}, k\right)\right|_{\tau(t) \in I_{k}}=\dot{V}_{1}\left(x_{t}\right)+\dot{V}_{2}\left(x_{t}\right)+\dot{V}_{3}\left(x_{t}, k\right)+\dot{V}_{4}\left(x_{t}\right)
$$

where

$$
\dot{V}_{1}\left(x_{t}\right)=2 \epsilon^{\mathrm{T}}(t) \mathcal{P} \dot{\epsilon}(t)=\xi^{\mathrm{T}}(t) \Psi_{1} \xi(t)
$$

The derivative of the second term of the $V_{2}\left(x_{t}\right)$ is derived as

$$
\begin{aligned}
\frac{d}{d t}( & \left.\sum_{i=1}^{q-1} \int_{t-\tau_{i}}^{t-\tau_{i-1}} x^{\mathrm{T}}(t) Q_{i} x(t) \mathrm{d} s\right)=\sum_{i=1}^{q-1}\left(x^{\mathrm{T}}\left(t-\tau_{i-1}\right) Q_{i} x\left(t-\tau_{i-1}\right)-x^{\mathrm{T}}\left(t-\tau_{i}\right) Q_{i} x\left(t-\tau_{i}\right)\right) \\
= & x^{\mathrm{T}}\left(t-\tau_{0}\right) Q_{1} x\left(t-\tau_{0}\right)+x^{\mathrm{T}}\left(t-\tau_{1}\right)\left(Q_{2}-Q_{1}\right) x\left(t-\tau_{1}\right)+x^{\mathrm{T}}\left(t-\tau_{2}\right)\left(Q_{3}-Q_{2}\right) x\left(t-\tau_{2}\right) \ldots \\
& +x^{\mathrm{T}}\left(t-\tau_{q-2}\right)\left(Q_{m}-Q_{m-1}\right) x\left(t-\tau_{q-2}\right)-x^{\mathrm{T}}\left(t-\tau_{q-1}\right) Q_{m} x\left(t-\tau_{q-1}\right) \\
= & {\left[\begin{array}{c}
x\left(t-\tau_{0}\right) \\
x\left(t-\tau_{1}\right) \\
\vdots \\
x\left(t-\tau_{q-2}\right) \\
x\left(t-\tau_{q-1}\right)
\end{array}\right]\left[\begin{array}{ccccc}
Q_{1} & 0 & \cdots & \cdots & 0 \\
* & Q_{2}-Q_{1} & 0 & \cdots & \vdots \\
* & * & \ddots & 0 & \vdots \\
* & * & * & Q_{m}-Q_{m-1} & 0 \\
* & * & * & * & -Q_{m}
\end{array}\right]\left[\begin{array}{c}
x\left(t-\tau_{0}\right) \\
x\left(t-\tau_{1}\right) \\
\vdots \\
x\left(t-\tau_{q-2}\right) \\
x\left(t-\tau_{q-1}\right)
\end{array}\right] }
\end{aligned}
$$

Thus,

$$
\begin{aligned}
& \dot{V}_{2}\left(x_{t}\right) \leq x^{\mathrm{T}}(t) \widetilde{Q} x(t)-(1-\mu) x^{\mathrm{T}}(t-\tau(t)) \widetilde{Q} x(t-\tau(t)) \\
&+\left[\begin{array}{c}
x\left(t-\tau_{0}\right) \\
x\left(t-\tau_{1}\right) \\
\vdots \\
x\left(t-\tau_{q-2}\right) \\
x\left(t-\tau_{q-1}\right)
\end{array}\right]\left[\begin{array}{ccccc}
Q_{1} & 0 & \cdots & \cdots & 0 \\
* & Q_{2}-Q_{1} & 0 & \cdots & \vdots \\
* & * & \ddots & 0 & \vdots \\
* & * & * & Q_{m}-Q_{m-1} & 0 \\
* & * & * & * & -Q_{m}
\end{array}\right]\left[\begin{array}{c}
x\left(t-\tau_{0}\right) \\
x\left(t-\tau_{1}\right) \\
\vdots \\
x\left(t-\tau_{q-2}\right) \\
x\left(t-\tau_{q-1}\right)
\end{array}\right] \\
&=\xi^{\mathrm{T}}(t) \Psi_{2} \xi(t)
\end{aligned}
$$


The derivative of $V_{3}\left(x_{t}, k\right)$ is deduced as

$$
\dot{V}_{3}\left(x_{t}\right)=\dot{x}^{\mathrm{T}}(t)\left(\sum_{i=1}^{q-1} \delta_{i} \alpha^{q-i} Z_{i}\right) \dot{x}(t)-\sum_{i=1}^{q-1} \delta_{i} \int_{t-\tau_{i}}^{t-\tau_{i-1}} \dot{x}^{\mathrm{T}}(s) Z_{i} \dot{x}(s) \mathrm{d} s
$$

For the case of $\tau(t) \in I_{k}(1 \leq k \leq m)$, the second term in (19) is deduced as follows

$$
\begin{aligned}
& -\sum_{i=1}^{q-1} \delta_{i} \int_{t-\tau_{i}}^{t-\tau_{i-1}} \dot{x}^{\mathrm{T}}(s) Z_{i} \dot{x}(s) \mathrm{d} s \\
& \quad=-\sum_{i=1, i \neq k}^{q-1} \delta_{i} \int_{t-\tau_{i}}^{t-\tau_{i-1}} \dot{x}^{\mathrm{T}}(s) Z_{i} \dot{x}(s) \mathrm{d} s-\delta_{k} \int_{t-\tau_{k}}^{t-\tau_{k-1}} \dot{x}^{\mathrm{T}}(s) Z_{k} \dot{x}(s) \mathrm{d} s
\end{aligned}
$$

Applying Lemma 2 to deal with (20), it is obtained

$$
-\sum_{i=1, i \neq k}^{q-1} \delta_{i} \int_{t-\tau_{i}}^{t-\tau_{i-1}} \dot{x}^{\mathrm{T}}(s) Z_{i} \dot{x}(s) \mathrm{d} s \leq \sum_{i=1, i \neq k}^{q-1} \varpi_{3 i}^{\mathrm{T}}(t) \Omega_{3} \varpi_{3 i}(t)
$$

where $\varpi_{3 i}(t)=\left[x^{\mathrm{T}}\left(t-\tau_{i-1}\right) x^{\mathrm{T}}\left(t-\tau_{i}\right) \frac{1}{\delta_{i}} \int_{t-\tau_{i}}^{t-\tau_{i-1}} x^{\mathrm{T}}(s) \mathrm{d} s\right]^{\mathrm{T}}$.

In the case of $i=k$, applying Jensen's inequality and the extended ERCC in Lemma 3, it is given as,

$$
\begin{aligned}
& -\left(\tau_{k}-\tau_{k-1}\right) \int_{t-\tau_{k}}^{t-\tau_{k-1}} \dot{x}^{\mathrm{T}}(s) Z_{k} \dot{x}(s) \mathrm{d} s \\
& =-\left(\tau_{k}-\tau_{k-1}\right)\left(\int_{t-\tau(t)}^{t-\tau_{k-1}} \dot{x}^{\mathrm{T}}(s) Z_{k} \dot{x}(s) \mathrm{d} s+\int_{t-\tau_{k}}^{t-\tau(t)} \dot{x}^{\mathrm{T}}(s) Z_{k} \dot{x}(s) \mathrm{d} s\right) \\
& \leq-\frac{\left(\tau_{k}-\tau_{k-1}\right)}{\left(\tau(t)-\tau_{k-1}\right)}\left(\int_{t-\tau(t)}^{t-\tau_{k-1}} \dot{x}^{\mathrm{T}}(s) \mathrm{d} s\right) Z_{k}\left(\int_{t-\tau(t)}^{t-\tau_{k-1}} \dot{x}(s) \mathrm{d} s\right) \\
& -\frac{\left(\tau_{k}-\tau_{k-1}\right)}{\left(\tau_{k}-\tau(t)\right)}\left(\int_{t-\tau_{k}}^{t-\tau(t)} \dot{x}^{\mathrm{T}}(s) \mathrm{d} s\right) Z_{k}\left(\int_{t-\tau_{k}}^{t-\tau(t)} \dot{x}(s) \mathrm{d} s\right) \\
& =-\frac{\left(\tau_{k}-\tau_{k-1}\right)}{\left(\tau(t)-\tau_{k-1}\right)}\left[\begin{array}{l}
x\left(t-\tau_{k-1}\right) \\
x(t-\tau(t))
\end{array}\right]^{\mathrm{T}}\left[\begin{array}{ll}
Z_{k}-Z_{k} \\
* & Z_{k}
\end{array}\right]\left[\begin{array}{l}
x\left(t-\tau_{k-1}\right) \\
x(t-\tau(t))
\end{array}\right] \\
& -\frac{\left(\tau_{k}-\tau_{k-1}\right)}{\left(\tau_{k}-\tau(t)\right)}\left[\begin{array}{l}
x(t-\tau(t)) \\
x\left(t-\tau_{k}\right)
\end{array}\right]^{\mathrm{T}}\left[\begin{array}{cc}
Z_{k}-Z_{k} \\
* & Z_{k}
\end{array}\right]\left[\begin{array}{l}
x(t-\tau(t)) \\
x\left(t-\tau_{k}\right)
\end{array}\right] \\
& \leq-\eta_{0}^{\mathrm{T}}(t)\left[\begin{array}{lll}
Z_{k}-Z_{k}+J & -J \\
* & 2 Z_{k}-J^{\mathrm{T}}-J-Z_{k}+J \\
* & * & Z_{k}
\end{array}\right] \eta_{0}(t)
\end{aligned}
$$

where $\eta_{0}(t)=\left[x^{\mathrm{T}}\left(t-\tau_{k-1}\right) x^{\mathrm{T}}(t-\tau(t)) x^{\mathrm{T}}\left(t-\tau_{k}\right)\right]^{\mathrm{T}}$.

Then , it follows from (19-22) that

$$
\dot{V}_{3}\left(x_{t}, k\right) \leq \dot{x}^{\mathrm{T}}(t)\left(\sum_{i=1}^{q-1} \delta_{i} \alpha^{q-i} Z_{i}\right) \dot{x}(t)+\xi^{\mathrm{T}}(t) \Psi_{3 k} \xi(t)
$$


The derivative of $V_{4}\left(x_{t}\right)$ is presented as

$$
\begin{aligned}
\dot{V}_{4}\left(x_{t}\right)= & \dot{x}^{\mathrm{T}}(t)\left(\sum_{i=1}^{q-1} \frac{1}{4} \tau_{i-1}^{4} R_{2 i}+\sum_{i=1}^{q-1} \frac{1}{4}\left(\tau_{i}^{2}-\tau_{i-1}^{2}\right)^{2} R_{3 i}\right) \dot{x}(t) \\
& -\sum_{i=1}^{q-1} \frac{\tau_{i-1}^{2}}{2} \int_{-\tau_{i-1}}^{0} \int_{t+\theta}^{t} \dot{x}^{\mathrm{T}}(s) R_{2 i} \dot{x}(s) \mathrm{d} s \mathrm{~d} \theta \\
& -\sum_{i=1}^{q-1} \frac{\tau_{i}^{2}-\tau_{i-1}^{2}}{2} \int_{-\tau_{i}}^{-\tau_{i-1}} \int_{t+\theta}^{t} \dot{x}^{\mathrm{T}}(s) R_{3 i} \dot{x}(s) \mathrm{d} s \mathrm{~d} \theta
\end{aligned}
$$

By using Lemma 1, the last two terms of (24) are deduced as

$$
\begin{aligned}
& -\sum_{i=1}^{q-1} \frac{\tau_{i-1}^{2}}{2} \int_{-\tau_{i-1}}^{0} \int_{t+\theta}^{t} \dot{x}^{\mathrm{T}}(s) R_{2 i} \dot{x}(s) \mathrm{d} s \mathrm{~d} \theta \\
& \quad \leq \sum_{i=1}^{q-1}\left[\begin{array}{l}
\tau_{i-1} x(t) \\
\int_{t-\tau_{i-1}}^{t} x(s) \mathrm{d} s
\end{array}\right]^{\mathrm{T}}\left[\begin{array}{ll}
-R_{2 i} & R_{2 i} \\
* & -R_{2 i}
\end{array}\right]\left[\begin{array}{l}
\tau_{i-1} x(t) \\
\int_{t-\tau_{i-1}}^{t} x(s) \mathrm{d} s
\end{array}\right] \\
& -\sum_{i=1}^{q-1} \frac{\tau_{i}^{2}-\tau_{i-1}^{2} \int_{-\tau_{i}}^{-\tau_{i-1}} \int_{t+\theta}^{t} \dot{x}^{\mathrm{T}}(s) R_{3 i} \dot{x}(s) \mathrm{d} s \mathrm{~d} \theta}{2} \\
& \quad \leq \sum_{i=1}^{q-1}\left[\begin{array}{ll}
\left(\tau_{i}-\tau_{i-1}\right) x(t) \\
\int_{t-\tau_{i}}^{t-\tau_{i-1}} x(s) \mathrm{d} s
\end{array}\right]^{\mathrm{T}}\left[\begin{array}{ll}
-R_{3 i} & R_{3 i} \\
* & -R_{3 i}
\end{array}\right]\left[\begin{array}{l}
\left(\tau_{i}-\tau_{i-1}\right) x(t) \\
\int_{t-\tau_{i}}^{t-\tau_{i-1}} x(s) \mathrm{d} s
\end{array}\right] \\
& \quad \leq \sum_{i=1}^{q-1}\left(\tau_{i}-\tau_{i-1}\right)^{2}\left[\begin{array}{l}
x(t) \\
\frac{1}{\delta_{i}} \int_{t-\tau_{i}}^{t-\tau_{i-1}} x(s) \mathrm{d} s
\end{array}\right]^{\mathrm{T}}\left[\begin{array}{l}
-R_{3 i} R_{3 i} \\
*
\end{array}\right]\left[\begin{array}{c}
x(t) \\
\frac{1}{\delta_{i}} \int_{t-\tau_{i}}^{t-\tau_{i-1}} x(s) \mathrm{d} s
\end{array}\right]
\end{aligned}
$$

Thus (24) implies that

$$
\dot{V}_{4}\left(x_{t}\right) \leq \dot{x}^{\mathrm{T}}(t)\left(\sum_{i=1}^{q-1} \frac{1}{4} \tau_{i-1}^{4} R_{2 i}+\sum_{i=1}^{q-1} \frac{1}{4}\left(\tau_{i}^{2}-\tau_{i-1}^{2}\right)^{2} R_{3 i}\right) \dot{x}(t)+\xi^{\mathrm{T}}(t) \Psi_{4} \xi(t)
$$

Referring to (5), for any scalars $\lambda_{1} \geq 0, \lambda_{2} \geq 0$, the nonlinear perturbations can be derived as

$$
\begin{aligned}
& 0 \leq \lambda_{1}\left(\gamma^{2} x^{\mathrm{T}}(t) F^{\mathrm{T}} F x(t)-f^{\mathrm{T}} f\right)=\xi^{\mathrm{T}}(t) \Psi_{51} \xi(t) \\
& 0 \leq \lambda_{2}\left(\beta^{2} x^{\mathrm{T}}(t-\tau(t)) G^{\mathrm{T}} G x(t-\tau(t))-g^{\mathrm{T}} g\right)=\xi^{\mathrm{T}}(t) \Psi_{52} \xi(t)
\end{aligned}
$$

According to the system in (1), with $\widehat{N}_{1}$ and $\widehat{N}_{2}$ are defined as $\widehat{N}_{1}=\sum_{l=1}^{r} h_{l}(t) \widehat{N}_{1 l}$ and $\widehat{N}_{2}=\sum_{l=1}^{r} h_{l}(t) \widehat{N}_{2 l}$, and $\widehat{N}_{1 l}, \widehat{N}_{2 l}$ are constant matrices. Then it is given as

$$
\begin{aligned}
0= & 2\left[\dot{x}^{\mathrm{T}}(t) \widehat{N}_{1}+x^{\mathrm{T}}(t) \widehat{N}_{2}\right]\left[A_{l} x(t)+B_{l} x(t-\tau(t))+C_{l} f(x(t), t)\right. \\
& \left.+D_{l} g(x(t-\tau(t)), t)-\dot{x}(t)\right] \\
= & \xi^{\mathrm{T}}(t) \Psi_{l, 6} \xi(t)
\end{aligned}
$$


Therefore, the following inequality holds

$$
\left.\dot{V}\left(x_{t}, k\right)\right|_{\tau(t) \in I_{k}} \leq \sum_{l=1}^{r} h_{l}(t) \xi^{\mathrm{T}}(t) \Psi_{k, l} \xi(t)
$$

Using the augmented vector (11) with the simplification expression (10), the T-S fuzzy system (2) is represented as

$$
0=\sum_{l=1}^{r} h_{l}(t) \Gamma_{l} \xi(t)
$$

where $\Gamma_{l}$ is defined in Theorem 1.

Hence, the asymptotic stability condition for the T-S fuzzy system (2) with interval time-varying delays and nonlinear perturbations is expressed as

$$
\begin{array}{r}
\sum_{l=1}^{r} h_{l}(t) \xi^{\mathrm{T}}(t) \Psi_{k, l} \xi(t)<0 \\
\text { subject to }: 0=\sum_{i=1}^{r} h_{l}(t) \Gamma_{l} \xi(t)
\end{array}
$$

Consequently, by means of the Lemma 4 , there exists a matrix $\mathcal{Y}$ with appropriate dimensions such that the (31) is equivalent to

$$
\sum_{l=1}^{r} h_{l}(t) \xi^{\mathrm{T}}(t)\left[\Psi_{k, l}+\operatorname{He}\left(\mathcal{Y} \Gamma_{l}\right)\right] \xi(t)<0
$$

As a result, the derivatives of the newly proposed Lyapunov functionals is deduced as $\left.\dot{V}\left(x_{t}, k\right)\right|_{\tau(t) \in I_{k}}<0$. It means $\left.\dot{V}\left(x_{t}, k\right)\right|_{\tau(t) \in I_{k}}<\rho\|x(t)\|^{2}$ for sufficiently small $\rho>0$. Hence the T-S fuzzy system in (2) is globally asymptotically stable. This completes the proof.

Remark 2 For the absence of perturbation, that is $C(t)=0, D(t)=0$, then the T-S fuzzy system (2) is simplified as

$$
\begin{aligned}
& \dot{x}(t)=A(t) x(t)+B(t) x(t-\tau(t)), \quad t \geq 0 \\
& x(t)=\varphi(t), \quad t \in\left[-\tau_{b}, 0\right]
\end{aligned}
$$

This system has been widely studied Zhao et al. (2009), Wu et al. (2011), Zhang et al. (2015b). The stability criterion for the system is stated below.

Theorem 2 Given a positive integer $m$, and $\delta_{i}=\alpha^{q-i}$. Consider (3) with time-varying delay satisfying

Case 1 The system (33) is asymptotically stable if there exist symmetric positive definite matrices $Z_{i}, Q_{i}, \widetilde{Q}, R_{2 i}, R_{3 i} \in \mathbb{R}^{n \times n}(i=1,2 \ldots, m), \quad \mathcal{P}=\left[P_{i j}\right]_{(m+1) \times(m+1)}$ 
$\in \mathbb{R}^{(m+1) n \times(m+1) n}$, symmetric matrices $W_{1}, W_{3} \in \mathbb{R}^{3 n \times 3 n}$, and $J \in \mathbb{R}^{n \times n}$, matrices $W_{2} \in \mathbb{R}^{3 n \times 3 n}, N_{1}, N_{2} \in \mathbb{R}^{3 n \times n}$, and $\mathcal{Y} \in \mathbb{R}^{(3 m+4) n \times n}$, such that the following LMIs hold

$$
\begin{aligned}
& {\left[\begin{array}{lll}
W_{1} & W_{2} & N_{1} \\
* & W_{3} & N_{2} \\
* & * & Z_{i}
\end{array}\right] \geq 0} \\
& \widetilde{\Psi}_{k, l}+\operatorname{He}\left(\mathcal{Y} \Gamma_{l}\right)<0, \quad l=1,2, \ldots, r
\end{aligned}
$$

where $\quad \Gamma_{l}=A_{l} e_{2}^{\mathrm{T}}+B_{l} e_{m+4}^{\mathrm{T}}-e_{1}^{\mathrm{T}}, \quad \widetilde{\Psi}_{k, l}=\Psi_{1}+\Psi_{2}+\Psi_{3 k}+\Psi_{4}+\widetilde{\Psi}_{l 6}+e_{1} \mathcal{Z} e_{1}^{\mathrm{T}}, \quad$ and $\Psi_{1}, \Psi_{2}, \Psi_{3 k}, \Psi_{4}, \mathcal{Z}$ have been defined in Theorem $1 . \widetilde{\Psi}_{l 6}$ can be deduced by removing the perturbed elements $C_{l} f(x(t), t)$ and $D_{l} g(x(t-\tau(t)), t)$ in (28).

Proof The same Lyapunov-Krasovskii functional candidate (14) for system (33) is selected for stability analysis. The augment vector (11) is modified as

$$
\widetilde{\xi}(t)=\left[\dot{x}^{\mathrm{T}}(t), x^{\mathrm{T}}(t), x^{\mathrm{T}}\left(t-\tau_{0}\right), \eta^{\mathrm{T}}(t), x^{\mathrm{T}}(t-\tau(t)), \eta_{1}^{\mathrm{T}}(t), \eta_{2}^{\mathrm{T}}(t)\right]^{\mathrm{T}}
$$

where $\eta(t), \eta_{1}(t)$ and $\eta_{2}(t)$ are defined in Theorem 1 . Then following the similar process of the proof of Theorem 1, the asymptotic stability condition for the T-S system (33) is equivalent to

$$
\sum_{l=1}^{r} h_{l} \widetilde{\xi}^{\mathrm{T}}(t)\left[\widetilde{\Psi}_{k}+\operatorname{He}\left(\mathcal{Y} \Gamma_{l}\right)\right] \widetilde{\xi}(t)<0
$$

This completes the proof.

Corollary 1 Given a positive integer $m$, and $\delta_{i}=\alpha^{q-i}$. Considering $\tau(t)$ is a continuous function in (4). Then the system (2) is asymptotically stable if there exist symmetric positive definite matrices $Z_{i}, Q_{i}, R_{2 i}, R_{3 i} \in \mathbb{R}^{n \times n}(i=1,2 \ldots, m)$, $\mathcal{P}=\left[P_{i j}\right]_{(m+1) \times(m+1)} \in \mathbb{R}^{(m+1) n \times(m+1) n}$, symmetric matrices $W_{1}, W_{3} \in \mathbb{R}^{3 n \times 3 n}$, and $J \in \mathbb{R}^{n \times n}$, matrices $W_{2} \in \mathbb{R}^{3 n \times 3 n}, N_{1}, N_{2} \in \mathbb{R}^{3 n \times n}$, and $\mathcal{Y} \in \mathbb{R}^{(3 m+6) n \times n}$, such that the following LMIs hold

$$
\begin{aligned}
& {\left[\begin{array}{ccc}
W_{1} & W_{2} & N_{1} \\
* & W_{3} & N_{2} \\
* & * & \widetilde{Z}_{3}
\end{array}\right] \geq 0} \\
& \widehat{\Psi}_{k, l}+\operatorname{He}\left(\mathcal{Y} \Gamma_{l}\right)<0, \quad l=1,2, \ldots r
\end{aligned}
$$

where $\widehat{\Psi}_{k, l}$ is deduced from $\Psi_{k, l}$ by replacing $\Psi_{2}$ as

$$
\widehat{\Psi}_{2}=\left[\begin{array}{c}
e_{3}^{\mathrm{T}} \\
e_{4}^{T} \\
\vdots \\
e_{m+2}^{\mathrm{T}} \\
e_{m+3}^{\mathrm{T}}
\end{array}\right]^{\mathrm{T}}\left[\begin{array}{ccccc}
Q_{1} & 0 & \cdots & \cdots & 0 \\
* & Q_{2}-Q_{1} & 0 & \cdots & \vdots \\
* & * & \ddots & 0 & \vdots \\
* & * & * & Q_{m}-Q_{m-1} & 0 \\
* & * & * & * & -Q_{m}
\end{array}\right]\left[\begin{array}{c}
e_{3}^{\mathrm{T}} \\
e_{4}^{\mathrm{T}} \\
\vdots \\
e_{m+2}^{\mathrm{T}} \\
e_{m+3}^{\mathrm{T}}
\end{array}\right]
$$


$\Gamma_{l}$ are defined in Theorem 1.

Proof For the T-S fuzzy system (2) with interval time-varying delays, modify the Lyapunov functionals (14) by setting $\widetilde{Q}=0$, i.e., $\widehat{V}_{2}\left(x_{t}\right)=\sum_{i=1}^{q-1} \int_{t-\tau_{i}}^{t-\tau_{i-1}} x^{\mathrm{T}}(t) Q_{i} x(t) \mathrm{d} s$. Then following the similar process of the proof of Theorem 1 , the asymptotic stability condition for the T-S system (2) is equivalent to

$$
\sum_{l=1}^{r} h_{l} \xi^{\mathrm{T}}(t)\left[\widehat{\Psi}_{k, l}+\operatorname{He}\left(\mathcal{Y} \Gamma_{l}\right)\right] \xi(t)<0
$$

This completes the proof.

Remark 3 Both lower and upper bounds of the time-varying delay $\tau(t)$ are concerned in Cases 1 and 2. Actually, it is pointed out that Case 1 is a special case of Case 2, which means less conservative results can be obtained by using Case 1 instead of Case 2 in the case of a differentiable function of $\tau(t)$. Nonetheless, if $\tau(t)$ is not differentiable, Case 2 is able to overcome this issue Peng and Han (2011).

Remark 4 Considering a unit common ratio, i.e. $\alpha=1$, which means the length of each subinterval is equivalent. Then previous developed research works using the equivalent partition method Hui et al. (2015), Wang and Shen (2012), Zhao et al. (2009) can be considered as a special case of this proposed approach. Therefore, the developed partitioning method is more generalized.

\section{Numerical example}

In this section, numerical examples are conducted to investigate the stability of the $\mathrm{T}-\mathrm{S}$ fuzzy systems in (2) and (33).

Example 1 Consider the nominal T-S fuzzy systems (33) with the fuzzy rules described in Peng et al. (2011), Zeng et al. (2015b), Liu et al. (2010) as follows:

Rule 1 : If $z_{1}(t)$ is $\pm \pi / 2$, then $\dot{x}(t)=A_{1} x(t)+B_{1} x(t-\tau(t))$

Rule 2: If $z_{2}(t)$ is \pm 0 , then $\dot{x}(t)=A_{2} x(t)+B_{2} x(t-\tau(t))$

where the parameters widely discussed are given as,

$A_{1}=\left[\begin{array}{cc}-2.1 & 0.1 \\ -0.2 & -0.9\end{array}\right], B_{1}=\left[\begin{array}{cc}-1.1 & 0.1 \\ -0.2 & -0.9\end{array}\right], A_{2}=\left[\begin{array}{cc}-1.9 & 0 \\ -0.2 & -1.1\end{array}\right], B_{2}=\left[\begin{array}{cc}-0.9 & 0 \\ -1.1 & -1.2\end{array}\right]$.

In the Rules 1 and 2, the membership function are $h_{1}(z(t))=\frac{1}{1+\exp (-2 z(t))}$, $h_{2}(z(t))=1-h_{1}(z(t))$.

Considering the lower bound of the time-varying delay $\tau_{a}=0$, different values of delay derivative rate $\mu$ are selected to obtain the upper bound of $\tau_{b}$ for comparisons in Table 1.

In Table 1 , considering different values of $\mu$, the comparisons of the maximum upper bounds $\tau_{b}$ are given for $\tau_{a}=0$. According to results in Lian et al. (2016), it is clearly to show that for $\mu=0,0.1,0.5$ this proposed method can dramatically increase the 
Table 1 Upper bounds of $\tau_{b}$ for $\tau_{a}=0$ and different values of $\mu$

\begin{tabular}{lllll}
\hline Methods & $\boldsymbol{\mu}=\mathbf{0}$ & $\boldsymbol{\mu}=\mathbf{0 . 1}$ & $\boldsymbol{\mu}=\mathbf{0 . 5}$ & Unknown \\
\hline Liu et al. (2010) & 3.30 & 2.65 & 1.50 & 0.79 \\
Zeng et al. (2015b) $(\mathrm{m}=3)$ & 4.37 & 3.41 & 1.95 & 1.77 \\
Lian et al. (2016) & 4.35 & 3.55 & 2.32 & - \\
Theorem 2 $(\mathrm{m}=3)$ & 4.75 & 4.06 & 3.18 & 1.98 \\
\hline
\end{tabular}

upper bound of the time varying delay when selecting the partitioning number $m=3$. Figure 2 illustrates that with respect to the newly conducted maximum value of $\tau_{b}$ the state response still converges to zero, which means the T-S fuzzy system (41) is globally asymptotically stable.

Considering $\tau(t)$ to be a continuous function, as it is given in (4), i.e., $\mu$ is unknown. Then upper bound of the $\tau_{b}$ in this proposed work is compared with some other research results shown in the right column of Table 1.

Referring to the simulation results in Table 1 , selecting $\mu=0.1, \tau_{b}=4.06$ and $\mu=0.5, \tau_{b}=3.18$ the state response of the T-S fuzzy system (41) is conducted in Fig. 2 .

Considering unknown $\mu$, Fig. 3 is shown with $\tau_{b}=1.98$.

In Figures 2, 3, simulation performance illustrates that under the maximum tolerant delay $\tau_{b}$ shown in Table 1 the T-S fuzzy system (41) is asymptotical stable.

Example 2 Consider the T-S fuzzy systems (2) in the presence of nonlinear perturbations with the fuzzy rules as follows:

Rule 1: If $z_{1}(t)$ is $\pm \pi / 2$, then

$$
\dot{x}(t)=A_{1} x(t)+B_{1} x(t-\tau(t))+C_{1} f(x(t), t)+D_{1} g(x(t-\tau(t)), t)
$$

Rule 2: If $z_{2}(t)$ is \pm 0 , then

$$
\dot{x}(t)=A_{2} x(t)+B_{2} x(t-\tau(t))+C_{2} f(x(t), t)+D_{2} g(x(t-\tau(t)), t)
$$

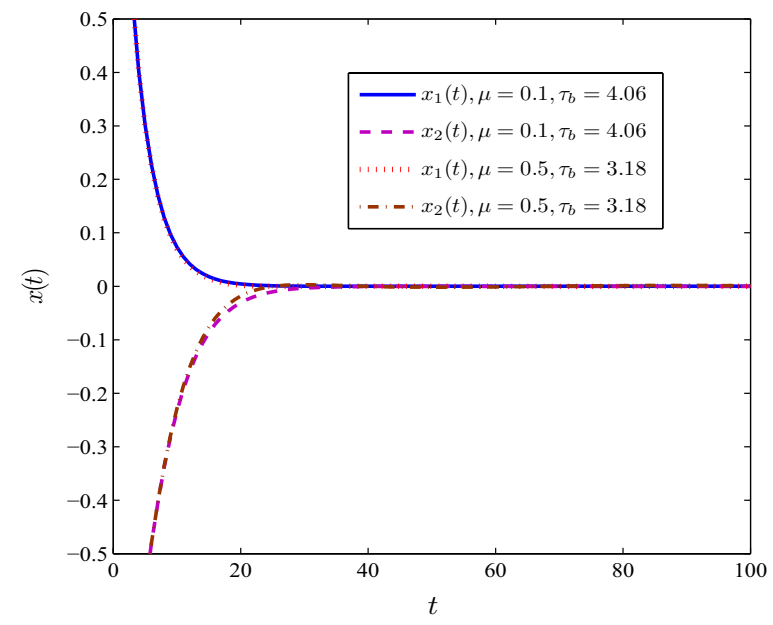

Fig. 2 The state response of system (41) 


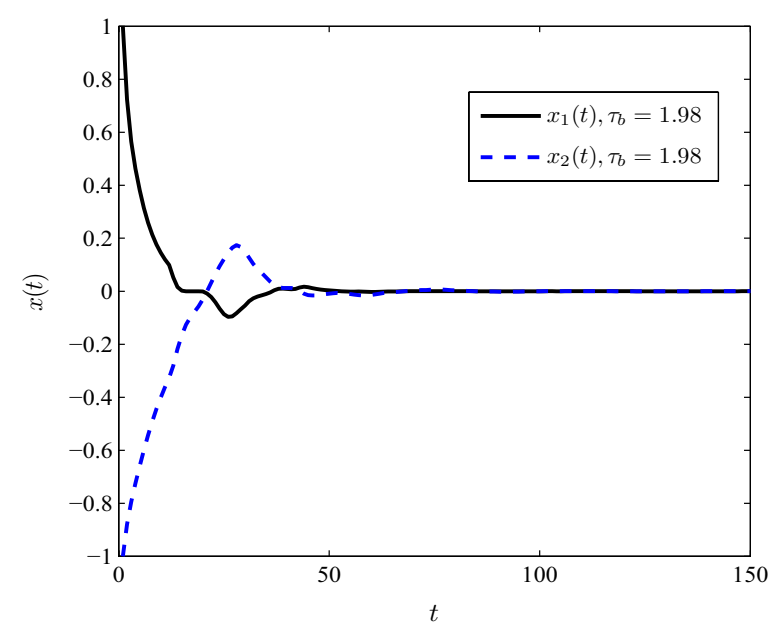

Fig. 3 The state response of system (41) with unknown $\mu$

Referring to the Assumption1, system parameters are given as,

$$
\begin{aligned}
& A_{1}=\left[\begin{array}{cc}
-2.1 & 0.1 \\
-0.2 & -0.9
\end{array}\right], B_{1}=\left[\begin{array}{cc}
-1.1 & 0.1 \\
-0.2 & -0.9
\end{array}\right], A_{2}=\left[\begin{array}{cc}
-1.9 & 0 \\
-0.2 & -1.1
\end{array}\right], B_{2}=\left[\begin{array}{cc}
-0.9 & 0 \\
-1.1 & -1.2
\end{array}\right], \\
& C_{1}=C_{2}=D_{1}=D_{2}=\left[\begin{array}{ll}
1 & 0 \\
0 & 1
\end{array}\right],
\end{aligned}
$$

and $\gamma=\beta=0.1$ In Rules 1 and 2, the membership function are $h_{1}(z(t))=\frac{1}{1+\exp (-2 z(t))}$, $h_{2}(z(t))=1-h_{1}(z(t))$.

For a given lower bound of $\tau_{a}=0$ in Theorem 1, considering different values of $\mu$ as well as the unknown $\mu$ in Corollary 1, the upper bounds of $\tau_{b}$ in this proposed work are obtained in Table 2.

In the presence of nonlinear perturbations, under a fixed value of delay derivative and the unknown $\mu$, the upper bound of delays are conducted in Table 2. It is shown that the proposed method works well in the perturbed T-S fuzzy system (2). By means of the simulation results in Table 2, selecting $\mu=0.5, \tau_{b}=1.94$ and unknown $\mu, \tau_{b}=1.72$ the state responses of the T-S system (41) are conducted in Figs. 4, 5.

Remark 5 By comparing with the results in Lian et al. (2016), Zeng et al. (2015b), Liu et al. (2010), less conservative results are obtained for the nominal T-S fuzzy system. Simulation results are conducted to demonstrate the remarkable improvements of the proposed method. The proposed geometric progression technique for delay partition can deal with the time-varying delayed T-S fuzzy systems with nonlinear perturbations with excellent stability criteria.

Remark 6 Tables 1 and 2 demonstrate that the maximum value of $\tau_{b}$ drops down when $\mu$ increases. In addition, the upper bound of time-varying delay $\tau(t)$ becomes bigger as soon as the partitioning segment gets finer. Figures 2, 3, 4 and 5 display that the convergence time of the state response rises up in the case of an unknown delay derivative $\mu$. 
Table 2 Upper bounds of $\tau_{b}$ for $\tau_{a}=0$ with different values of $\mu$ and unknown $\mu$

\begin{tabular}{lllll}
\hline Methods & $\boldsymbol{\mu}=\mathbf{0}$ & $\boldsymbol{\mu}=\mathbf{0 . 1}$ & $\boldsymbol{\mu}=\mathbf{0 . 5}$ & Unknown \\
\hline Theorem 1 $(\mathrm{m}=3)$ & 3.41 & 3.29 & 1.77 & 1.33 \\
Theorem 1 $(\mathrm{m}=4)$ & 4.39 & 4.28 & 1.94 & 1.72 \\
\hline
\end{tabular}

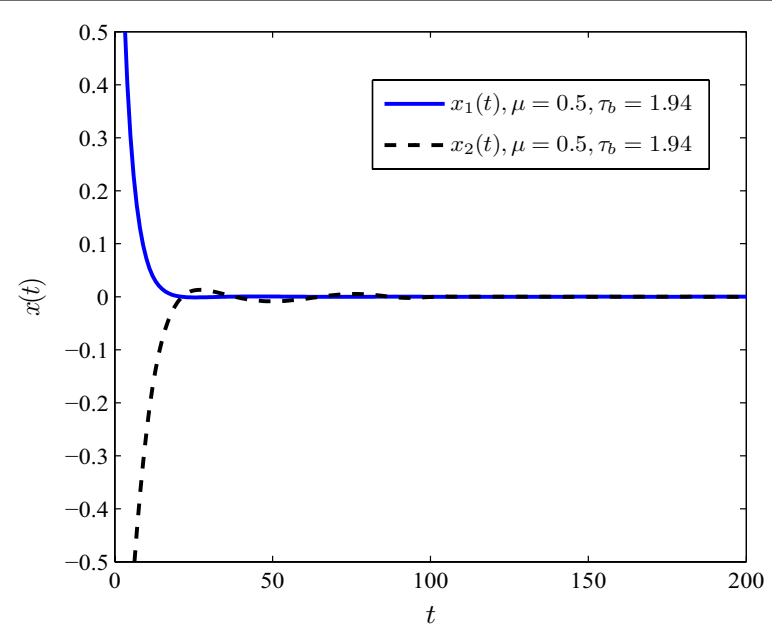

Fig. 4 The state response of system (42)

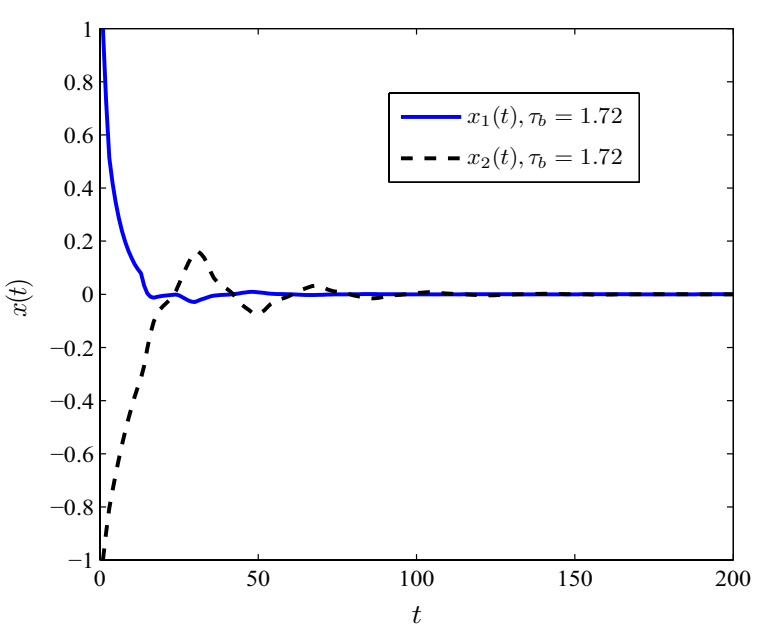

Fig. 5 The state response of system (42) with unknown $\mu$

\section{Conclusions}

In this paper, a novel delay partitioning method using the geometric sequence division is proposed for stability analysis of the perturbed T-S fuzzy system with interval timevarying delays. Recently developed inequalities and new modified Lyapunov functionals are introduced in this work. Numerical examples are given to demonstrate that less conservative results can be obtained in this design by comparing with some previously developed approaches. 


\section{Acknowledgements}

This work was partially supported by the Fundamental Research Funds for the Central Universities of China, Southwest University for Nationalities (2014NZYQN08).

\section{Competing interests}

The author declare that there is no competing interests.

Received: 15 April 2016 Accepted: 20 June 2016

Published online: 04 July 2016

\section{References}

Balasubramaniam P, Lakshmanan S, Rakkiyappan R (2012) Global robust stability criteria for T-S fuzzy systems with distributed delays and time delay in the leakage term. Iran J Fuzzy Syst 9(2):127-146

Chang W, Wang W-J (2015) $H_{\infty}$ fuzzy control synthesis for a large-scale system with a reduced number of Imis. IEEE Trans Fuzzy Syst 23(4):1197-1210

Chen H, Zhong S, Li M, Liu X, Adu-Gyamfi F (2016) Stability criteria for T-S fuzzy systems with interval time-varying delays and nonlinear perturbations based on geometric progression delay partitioning method. ISA Trans. doi:10.1016/j. isatra.2016.04.005

de Oliveira MC, Skelton RE (2001) Stability tests for constrained linear systems. In: Reza Moheimani SO (ed) Perspectives in robust control. Springer, London, pp 241-257

Gouaisbaut F, Peaucelle D (2006) Delay-dependent stability analysis of linear time delay systems. In: IFAC workshop on time delay system (TDS'06), vol. 6, L'Aquila, Italy

Gyurkovics É (2015) A note on wirtinger-type integral inequalities for time-delay systems. Automatica 61:44-46

Han Q-L (2003) Stability criteria for a class of linear neutral systems with time-varying discrete and distributed delays. IMA J Math Control Inf 20(4):371-386

Han Q-L (2005) A new delay-dependent stability criterion for linear neutral systems with norm-bounded uncertainties in all system matrices. Int J Syst Sci 36(8):469-475

Hui J-J, Kong X-Y, Zhang H-X, Zhou X (2015) Delay-partitioning approach for systems with interval time-varying delay and nonlinear perturbations. J Comput Appl Math 281:74-81

Lam J, Gao H, Wang C (2007) Stability analysis for continuous systems with two additive time-varying delay components. Syst Control Lett 56(1):16-24

Lian Z, He Y, Zhang C-K, Wu M (2016) Stability analysis for T-S fuzzy systems with time-varying delay via free-matrix-based integral inequality. Int J Control Autom Syst 14(1):21-28

Liu F, Wu M, He Y, Yokoyama R (2010) New delay-dependent stability criteria for T-S fuzzy systems with time-varying delay. Fuzzy Sets Syst 161(15):2033-2042

Park P, Ko JW, Jeong C (2011) Reciprocally convex approach to stability of systems with time-varying delays. Automatica 47(1):235-238

Park M, Kwon O, Park JH, Lee S, Cha E (2015) Stability of time-delay systems via wirtinger-based double integral inequality. Automatica 55:204-208

Peng C, Wen L-Y, Yang J-Q (2011) On delay-dependent robust stability criteria for uncertain T-S fuzzy systems with interval time-varying delay. Int J Fuzzy Syst 13(1):35-44

Peng C, Han Q-L (2011) Delay-range-dependent robust stabilization for uncertain T-S fuzzy control systems with interval time-varying delays. Inform Sci 181(19):4287-4299

Ramakrishnan K, Ray G (2011) Delay-range-dependent stability criterion for interval time-delay systems with nonlinear perturbations. Int J Autom Comput 8(1):141-146

Senthilkumar D, Mahanta C (2010) Identification of uncertain nonlinear systems for robust fuzzy control. ISA Trans 49(1):27-38

Seuret A, Gouaisbaut F (2013) Wirtinger-based integral inequality: application to time-delay systems. Automatica 49(9):2860-2866

Sun J, Liu G, Chen J, Rees D (2010) Improved delay-range-dependent stability criteria for linear systems with time-varying delays. Automatica 46(2):466-470

Takagi T, Sugeno M (1985) Fuzzy identification of systems and its applications to modeling and control. IEEE Trans Syst Man Cybernet SMC 14(1):116-132

Tanaka K, Hori T, Wang H (2003) A multiple lyapunov function approach to stabilization of fuzzy control systems. IEEE Trans Fuzzy Syst 11(4):582-589

Wang Z, Liu L, Shan Q-H, Zhang H (2015) Stability criteria for recurrent neural networks with time-varying delay based on secondary delay partitioning method. IEEE Trans Neural Netw Learn Syst 26(10):2589-2595

Wang C, Shen Y (2012) Delay partitioning approach to robust stability analysis for uncertain stochastic systems with interval time-varying delay. IET Control Theory Appl 6(7):875-883

Wu L, Su X, Shi P, Qiu J (2011) A new approach to stability analysis and stabilization of discrete-time T-S fuzzy time-varying delay systems. IEEE Trans Syst Man Cybernet B Cybernet 41(1):273-286

Yang J, Luo W, Cheng J, Wang Y (2015a) Further improved stability criteria for uncertain T-S fuzzy systems with interval time-varying delay by delay-partitioning approach. ISA Trans 58:27-34

Yang J, Luo W-P, Wang Y-H, Cheng J (2015b) Further improved stability criteria for uncertain T-S fuzzy systems with timevarying delay by $(m, N)$-delay-partitioning approach. ISA Trans 59:20-28

Zeng H-B, Park JH, Xia J-W, Xiao S-P (2014) Improved delay-dependent stability criteria for T-S fuzzy systems with timevarying delay. Appl Math Comput 235:492-501 
Zeng H, He Y, Wu M, She J (2015a) Free-matrix-based integral inequality for stability analysis of systems with time-varying delay. IEEE Trans Autom Control 60(10):2768-2772

Zeng H-B, He Y, Wu M, She J (2015b) New results on stability analysis for systems with discrete distributed delay. Automatica 60:189-192

Zhang W, Cai X-S, Han Z-Z (2010) Robust stability criteria for systems with interval time-varying delay and nonlinear perturbations. J Comput Appl Math 234(1):174-180

Zhang C, Deng F, Peng Y, Zhang B (2015a) Adaptive synchronization of cohen-grossberg neural network with mixed time-varying delays and stochastic perturbation. Appl Math Comput 269:792-801

Zhang Z, Lin C, Chen B (2015b) New stability and stabilization conditions for T-S fuzzy systems with time delay. Fuzzy Sets Syst 263:82-91

Zhao Y, Gao H, Lam J, Du B (2009) Stability and stabilization of delayed T-S fuzzy systems: a delay partitioning approach. IEEE Trans Fuzzy Syst 17(4):750-762

Submit your manuscript to a SpringerOpen ${ }^{\circ}$ journal and benefit from:

- Convenient online submission

\section{- Rigorous peer review}

- Immediate publication on acceptance

- Open access: articles freely available online

- High visibility within the field

- Retaining the copyright to your article

Submit your next manuscript at $\boldsymbol{\nabla}$ springeropen.com 